\title{
A 3-Dimensional Transnasal Endoscopic Journey Through the Paranasal Sinuses and Adjacent Skull Base: A Practical and Surgery-Oriented Perspective
}

\begin{abstract}
Andrea Bolzoni Villaret, MD*
Paolo Battaglia, MD $\ddagger$

Manfred Tschabitscher, MD, PhD§
\end{abstract}

Davide Mattavelli, MD*

Mario Turri-Zanoni, MD $\ddagger$

Paolo Castelnuovo, MD $\ddagger$

Piero Nicolai, MD*

*Department of Otorhinolaryngology, University of Brescia, Brescia, Italy; $\ddagger$ Department of Otorhinolaryngology, University of Insubria, Varese, Italy; §Department of Systematic Anatomy, Medical University of Wien, Wien, Austria

\section{Correspondence:}

Andrea Bolzoni Villaret, MD,

Department of OtorhinolaryngologyHead and Neck Surgery,

University of Brescia,

Spedali Civili of Brescia,

P.zza Spedali Civili, 1, 25123,

Brescia, Italy.

E-mail: dr.bolton@libero.it

Received, April 18, 2013.

Accepted, September 10, 2013.

Published Online, September 23, 2013.

Copyright $\odot 2013$ by the

Congress of Neurological Surgeons.

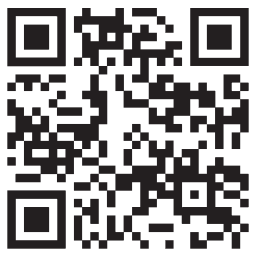

WHAT IS THIS BOX?

A QR Code is a matrix barcode readable by $Q R$ scanners, mobile phones with cameras, and smartphones. The QR Code above links to Supplemental Digital Content from this article.

An endoscopic approach through the transnasal corridor is currently the treatment of choice in the management of benign sinonasal tumors, cerebrospinal fluid leaks, and pituitary lesions. Moreover, this approach can be considered a valid option in the management of selected sinonasal malignancies extending to the skull base, midline meningiomas, parasellar lesions such as craniopharyngioma and Rathke cleft cyst, and clival lesions such as chordoma and ecchordosis.

Over the past decade, strict cooperation between otorhinolaryngologists and neurosurgeons and acquired surgical skills, together with high-definition cameras, dedicated instrumentation, and navigation systems, have made it possible to broaden the indications of endoscopic surgery. Despite these improvements, depth perception, as provided by the use of a microscope, was still lacking with this technology.

The aim of the present project is to reveal new perspectives in the endoscopic perception of the sinonasal complex and skull base thanks to 3-dimensional endoscopes, which are well suited to access and explore the endonasal corridor. In the anatomic dissection herein, this innovative device came across with sophisticated and longestablished fresh cadaver preparation provided by one of the most prestigious universities of Europe. The final product is a 3-dimensional journey starting from the nasal cavity, reaching the anterior, middle, and posterior cranial fossae, passing through the ethmoidal complex, paranasal sinuses, and skull base. Anatomic landmarks, critical areas, and tips and tricks to safely dissect delicate anatomic structures are addressed through audio comments, figures, and their captions.

KEY WORDS: Anatomy, Nasal cavity, Paranasal sinuses, Skull base, Three-dimensional endoscopy

Operative Neurosurgery 10:116-120, 2014

DOI: $10.1227 / \mathrm{NEU} .0000000000000172$

$\mathbf{T}$ he aim of this project is to use 3-dimensional (3-D) endoscopy to systematically explore the anatomy of the nose and paranasal sinuses as well as numerous skull base regions, ie, the sellar region, cavernous sinus, internal carotid artery, and floor of the middle cranial fossa.

The study was performed at the Department of Systematic Anatomy of the Medical School of

ABBREVIATIONS: CS, cavernous sinus; ET, eustachian tube; ICA, internal carotid artery; MMA, middle meningeal artery; $\mathbf{M N}$, mandibular nerve

Supplemental digital content is available for this article. Direct URL citations appear in the printed text and are provided in the HTML and PDF versions of this article on the journal's Web site (www.neurosurgery-online.com).
Vienna, Austria, and approved by the local Institutional Research Committee. Two fresh human cadaver heads were prepared for dissection after arterial injection of red-dyed liquid silicon. The heads had been checked for signs of craniofacial trauma, surgery, or any other condition potentially affecting the anatomy of the regions to be investigated. The endoscopic dissection was performed by using a 3-D endoscopy set (Visionsense, Ldt., Petach Tikva, Israel) consisting of $0^{\circ}$ and $30^{\circ}$ rigid endoscopes $(150 \mathrm{~mm}$ in length and $4.9 \mathrm{~mm}$ in diameter at their distal end). The set requires that passive 3-D glasses be worn to allow for binocular perception on a 3-D-ready screen. The transnasal endoscopic dissection, for its part, was performed with a complete set of dedicated instruments (Karl Storz, Germany). 
The resulting 10-minute pieces of stereoscopic footage were then arranged in the following order:

1. The nasal cavity and paranasal sinuses

2. The sphenoid sinus

3. The sellar and parasellar regions

4. The cavernous sinus, internal carotid artery, and infratemporal fossa

5. The vidian nerve, eustachian tube, V3, middle meningeal and internal carotid arteries.

Each section comprises a 3-D clip with a detailed step-by-step audio comment, a short text providing information as to all critical anatomic landmarks, and a triple picture showing 2-dimensional images and their legends, both of which are aimed at increasing viewer awareness.

By perceiving depth, volume, and distance, viewers will have a different perspective in evaluating the relationships between the anatomic structures being described. We designed and produced this multimedia 3-D endoscopy-based journey through nasal, paranasal, and skull base anatomy to share with the scientific community an all-new reviewing approach and identify opportunities and limitations posed by $3-\mathrm{D}$ endoscopy in this specific setting.

In some cases, a 3-D-based approach resulted in some slight peripheral image distortions during narrow space exploration, a problem that is likely to be even more significant during in vivo surgery owing to the presence of blood. Moreover, the $30^{\circ}$ device proved insufficient for some steps of the dissection, such as inspection of the maxillary sinus or when disassembling the frontal recess, for which $45^{\circ}$ or $70^{\circ}$ scopes would be better suited.

In contrast, the utility of 3-D endoscopy becomes fully apparent in larger workrooms and when a 2-nostril technique is applicable, such as in pituitary surgery or advanced skull base procedures. The negligible weight of the handle (98 g) and the absence of lateral wires make the device light and comfortable. In this perspective, a dependable cleaning system for its tip and appropriate holding device are both mandatory to maximize its potentials. In addition, the technique warrants comparative studies be performed to confirm the above-mentioned feedback and the feasibility of using the instrument in daily clinical practice be thoroughly assessed.

\section{THE NASAL CAVITY AND PARANASAL SINUSES}

The nasal cavity and the sphenoethmoidal complex can be considered as an anatomic pathway leading to the anterior, middle, and posterior cranial fossa (Figure 1A). This piece of footage shows the transnasal, anatomic dissection of the sinonasal compartment from a 3-D perspective. First, the ostiomeatal complex is approached, which is a common drainage area for the frontal, maxillary, and anterior ethmoid sinuses. The natural ostium of the maxillary sinus is exposed through partial inferior uncinectomy. To safely approach the frontal sinus, the frontal recess needs to be carefully disassembled by removing the cranial aspect of the uncinate process, the terminal recess, and the bulla ethmoidalis. Access is then gained to the posterior ethmoidal compartment by opening the second portion of the middle turbinate (basal lamella) laterally inserted on the lamina papyracea. At this stage, the lower edge of the superior turbinate is removed with cutting instruments, thus leading the surgeon directly to the natural ostium of the sphenoid sinus (Figure 1B).

During anteroposterior ethmoidectomy, the binocular stereoscopic view highlights the natural configuration of the ethmoid roof as it slopes down from its anterior to posterior portion, which in turn enables the surgeon to proceed without compromising the integrity of the skull base with clear identification of ethmoidal arteries (Figures 1B, C). In this way, the transethmoid corridor leading to the sphenoid sinus and sellar region will be obtained in a clean and safe manner, and the anatomic structures that surround it (the orbit laterally and the olfactory niche medially)
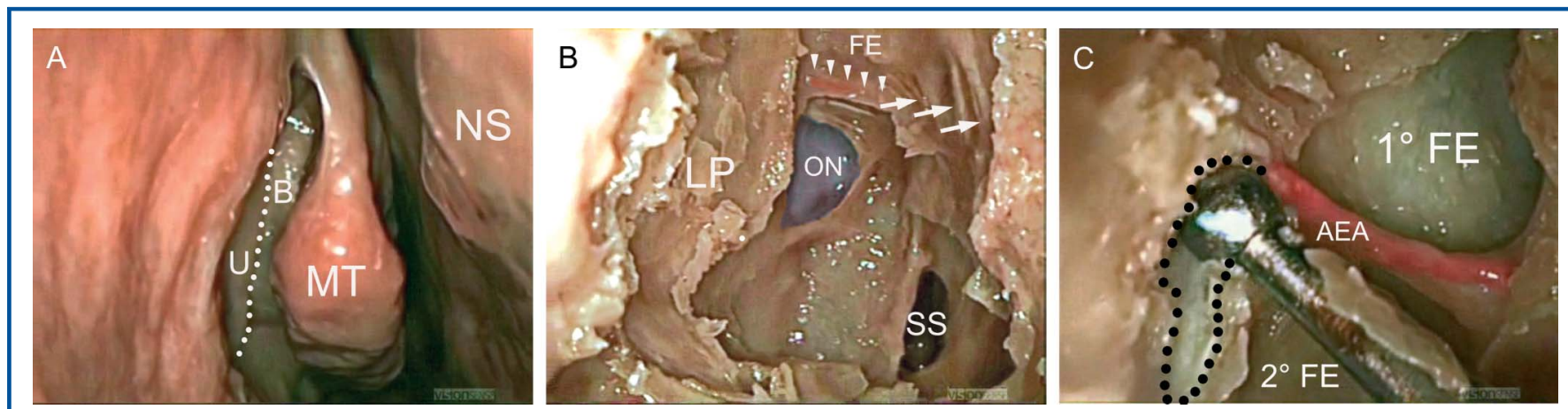

FIGURE 1. A, (00 minutes 35 seconds). Endoscopic view of the right nasal fossa through a $0^{\circ}$ instrument. The white dotted line runs on the free edge of the uncinate process $(U)$. B, (07 minutes 16 seconds). Postethmoidectomy endoscopic view of the right nasal fossa. An Onodi cell with the optic nerve (ON) inside is colored in blue. C, (10 minutes 12 seconds). Endoscopic view of the anterior left ethmoidal roof through a $30^{\circ}$ instrument: a blunt dissector is positioned through a skull base defect (black dotted line) on the dura of the olfactory cleft just where the anterior ethmoidal artery (AEA) gains access to the intradural space. NS, nasal septum; MT, middle turbinate; $B$, bulla ethmoidalis; LP, lamina papyracea; FE, foveola ethmoidalis; SS, sphenoid sinus; white arrowheads, posterior ethmoidal artery; white arrows, olfactory fibers along the superior turbinate; $1^{\circ} \mathrm{FE}$, first foveola; $2^{\circ} \mathrm{FE}$, second foveola. 
will be kept virtually intact (see Video 1, Supplemental Digital Content 1, http://youtu.be/NpuTJzhrHss).

\section{THE SPHENOID SINUS}

This piece of 3-D footage shows the anatomy of the main neural and vascular structures of the sphenoid and basisphenoid region (Figures 2A, B). In the crowded vascular network of this area, the nasal and septal branches of the sphenopalatine artery, the palatovaginal artery (Figure 2A), and the vidian artery and nerve (Figure 2B) can be easily identified. The vidian nerve can be used as a landmark for safe identification of the petrosal portion of the internal carotid artery (ICA) at the level of the anterior foramen lacerum. When the posterior wall of the pterygopalatine fossa has been reached, the maxillary nerve running through the foramen rotundum will become apparent (Figure 2B). Careful dissection of the pterygopalatine soft tissues will expose the bony maxillary strut separating the foramen rotundum from the superior orbital fissure.

The depth and volume perception is enhanced when we explore the ample surgical cavity obtained by removing the rostrum and intersphenoid septa. Here, the intrasphenoidal landmarks guiding the surgeon are the optic nerve, cavernous ICA, and the groove located in-between them (opticocarotid recess), the appearance of which may vary based on the degree of pneumatization of the optic strut and anterior clinoid process (Figure 2C). The sella and the upper portion of the clivus in the subsellar recess are visible along the midline. Finally, the sellar region is approached with exposure of the connective layers covering the pituitary gland. (see Video 2, Supplemental Digital Content 2, http://youtu.be/bn2Zd8T9hcE).

\section{THE SELLAR AND PARASELLAR REGIONS}

Removal of the bony walls and septations of the sphenoid sinus is strongly recommended. In fact, the variable degree of pneuma- tization of the sinus will sometimes make it hard to identify the landmarks leading to the sellar-parasellar region, ie, the optic nerve and the internal carotid artery bilaterally, the dural suspension ligaments connecting the sellar region with the cavernous sinuses, the dural fold at the level of tuberculum sellae, and the dura of the planum sphenoidalis (Figure 3A).

The suspension system of the pituitary gland is exposed after gently dissecting its capsule, as well as the arterial vessels supplying it: the inferior pituitary arteries and the meningohypophysial trunk, which has 3 terminal branches for the tentorium, the dura of the clival region, and the pituitary gland.

When the prechiasmatic cistern is accessed, the superior pituitary arteries will become visible (Figure 3B). Once the chiasmatic cistern has been opened, all the landmarks of the suprasellar region come to view, ie, pituitary stalk, optic chiasm, lamina terminalis, anterior cerebral arteries, and anterior communicating artery (Figure 3C).

Upon passing the dorsum sellae, the mid portion of the posterior cranial fossa can be explored. The basilar artery, anteroinferior cerebellar arteries, and abducent nerves will all become visible once the clivus has been removed and the prepontine dura has been cut through. The trigeminal roots, acoustic-facial bundle, vertebral and anteroinferior cerebellar arteries, as well as the roots of the glossopharyngeal, vagal, spinal, and hypoglossal nerves will all be visible from an inferolateral perspective. (see Video 3, Supplemental Digital Content 3, http://youtu.be/cmvUzD50EKk).

\section{THE CAVERNOUS SINUS, INTERNAL CAROTID ARTERY, AND INFRATEMPORAL FOSSA}

The cavernous sinus (CS) is the center of multiple venous pathways: intercavernous sinuses, ophthalmic veins, sylvian vein, emissary vein of the pterygoid plexus, foramen spinosum vein, and the superior and inferior petrous sinuses. Moreover, many venous
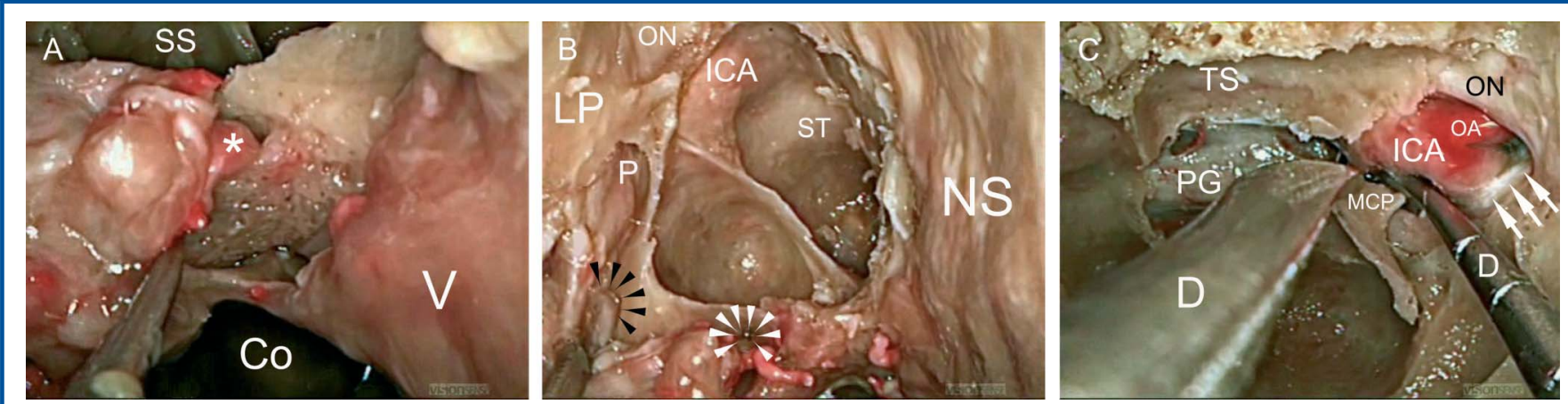

FIGURE 2. A, (01 minute 14 second). Blunt dissection of the right choana: the palatovaginal artery (white asterisk) is visible between the basisphenoid and the sphenoidal process of the palatine bone. B, (02 minute 57 seconds). The vidian canal (white arrowheads) and foramen rotondum (black arrowheads) are clearly visible. C, (05 minutes 47 seconds). Dissection of the left lateral wall of the sphenoid sinus: the median clinoid process (MCP) is removed and an atypical intracavernous origin of the ophthalmic artery $(O A)$ can be seen just below the optic nerve (ON); the white arrows point at the internal carotid artery's (ICA) proximal ring. SS, sphenoid sinus; Co, choana; V, vomer; LP, lamina papyracea; $P$, periorbit; ST, sella turcica; NS, nasal septum; TS, tuberculum sellae; $P G$, pituitary gland; $D$, dissector. 

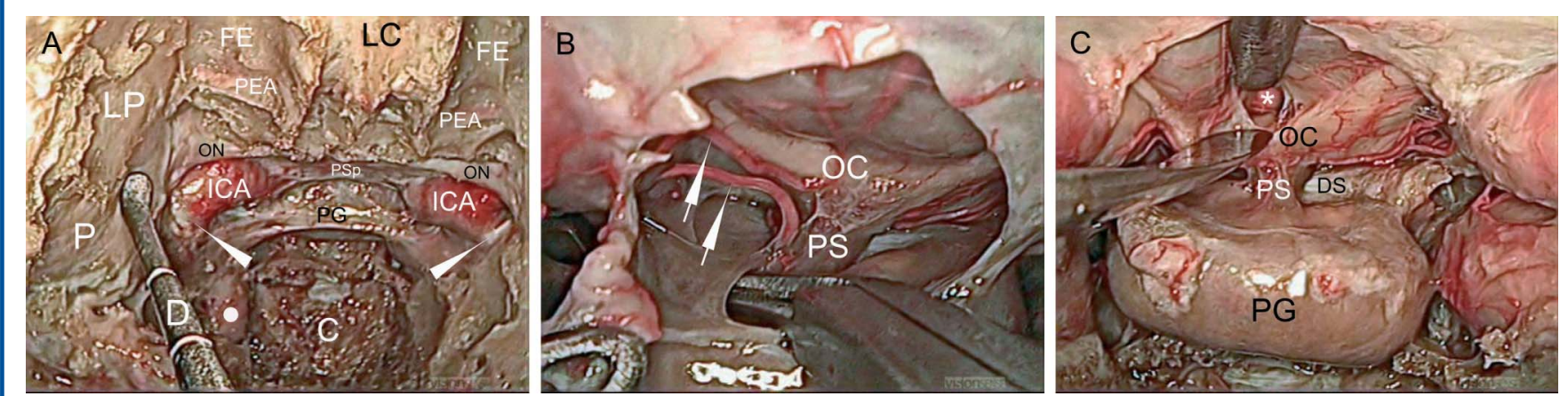

FIGURE 3. A, (00 minute 25 seconds). Bony substance is removed from the sellar floor and adjacent areas. A dissector (D) is positioned on the right periorbit (P). B, (03 minutes 46 seconds). The dura is opened at the level of tuberculum sellae, making the content of the prechiasmatic cistern visible. The tip of a curved scissor is then placed onto the porus of the sellar diaphragm. C, (05 minutes 17 seconds). The chiasmatic cistern is opened to expose the lamina terminalis and anterior cerebral arteries (asterisk). LC, lamina cribrosa; FE, fovea ethmoidalis; PEA, posterior ethmoidal artery; $O N$, optic nerve; ICA, internal carotid artery; PSp, planum sphenoidalis; PG, pituitary gland; LP, lamina papyracea; $C$, clivus; white dot, vertical portion of the cavernous internal carotid artery; white arrowheads, the proximal ring seen in a bilateral perspective; OC, optic chiasm; PS, pituitary stalk; white arrows, superior pituitary vascular pattern; DS, dorsum sellae.

plexuses are mutually connected: the carotid, foramen ovale, foramen rotondum, and the basilar plexus.

The abducens is the only nerve running through the CS not yet penetrating its lateral wall. The first branch of the trigeminal nerve is in close contact with the abducens on the anterior aspect of the CS. Once the plane connecting these 2 nerves has been identified, the trochlear and oculomotor nerves will be observed superiorly, and the second branch of the trigeminal nerve inferiorly (Figure 4A). The ICA enters the posterior aspect of the CS while exiting the carotid canal of the petrous bone below the petrolingual ligament (Figure $4 \mathrm{~B}$ ) and the trigeminal branches. Robust ligaments (proximal and distal rings) fix the ICA to the anterior clinoid process just before it gains access to the intradural compartment.

To reach the floor of the middle cranial fossa, the dissection must be extended into the pterygopalatine, pterygomaxillary, and infratemporal fossae. Once the posterior wall of the maxillary sinus has been opened, its vascular (maxillary artery and its branches) and neural structures (maxillary and vidian nerves) can be seen. The muscles of the masticatory space (from medial to lateral: the lateral pterygoid, temporalis, and masseter) will be exposed by removing the fatty tissue
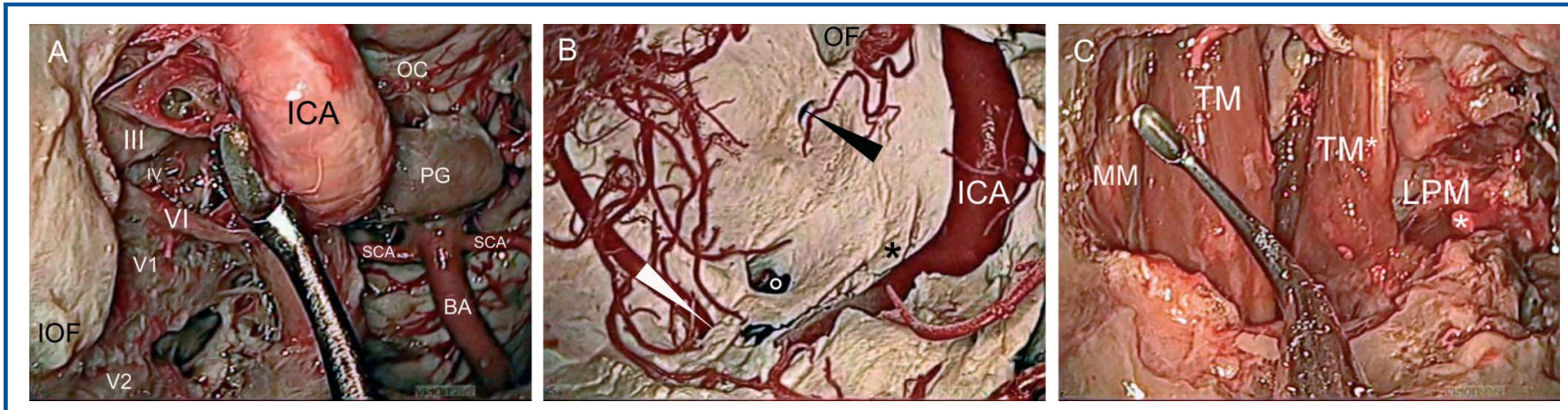

FIGURE 4. A, (01 minute 06 seconds). The content of the cavernous sinus becomes visible after its anteromedial walls have been removed. The ICA is medially displaced. B, (03 minutes 23 seconds). Posterior-to-anterior transcranial view of the middle cranial fossa. $\mathrm{C},(07$ minutes 04 seconds). Transnasal endoscopic view of the masticatory space and infratemporal fossa. A curette is in contact with the medial aspect of the right zygomatic arch where the masseter muscle (MM) is inserted. IOF, inferior orbital fissure; III, oculomotor nerve; IV, trochlear nerve; VI, abducens nerve; V1, first trigeminal branch; V2, second trigeminal branch; ICA, internal carotid artery; OC, optic chiasm; PG, pituitary gland; SCA, superior cerebellar artery; BA, basilar artery; OF, superior orbital fissure; white arrowhead, middle meningeal artery; black arrowhead, foramen rotondum; white circle, foramen ovale; black asterisk, lingula sphenoidalis; LPM, lateral pterygoid muscle; TM*, medial portion of the temporalis muscle proximally inserted in the infratemporal fossa; TM, lateral portion of the temporalis muscle proximally inserted in the temporal fossa; white asterisk, the stalk of the maxillary artery running through the pterygoid musculature. 

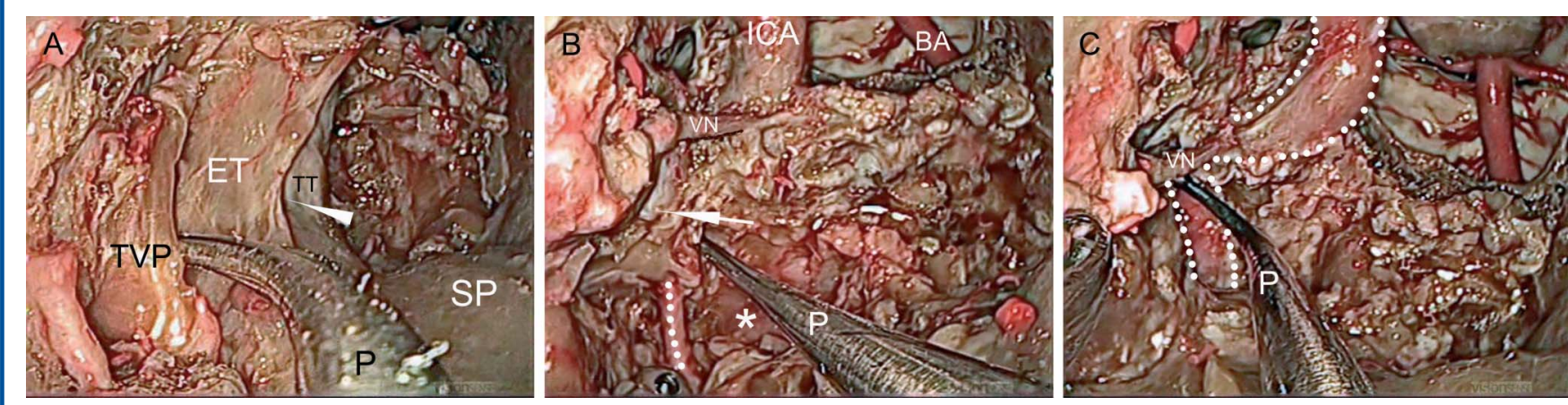

FIGURE 5. A, (01 minute 43 seconds). Right lateral wall of the nasopharynx after resection of the medial pterygoid plate: the probe (P) is positioned between the tensor veli palatini muscle (TVP) and the eustachian tube (ET); the arrowhead indicates the tube's natural ostium. B, (06 minutes 46 seconds). Once its cartilaginous component has been resected, the probe $(P)$ is positioned into the bony portion of the ET; the dotted line highlights the middle meningeal artery running through the foramen spinosum; the arrow points at the dura of the middle cranial fossa, and the white asterisk identifies the parapharyngeal portion of the internal carotid artery. C, $(08$ minutes 09 second). A probe (P) mimicking the orientation of the ET is now placed into the middle ear; the double dotted line outlines the course of the internal carotid artery from the cavernous portion down to the parapharyngeal space. TT, torus tubarius; SP, soft palate; VN, vidian nerve; ICA, internal carotid artery; BA, basilar artery.

(Figure 4C) (see Video 4, Supplemental Digital Content 4, http://youtu.be/eVxdvMo0omc).

\section{THE VIDIAN NERVE, EUSTACHIAN TUBE, V3, MIDDLE MENINGEAL AND INTERNAL CAROTID ARTERIES}

Dissecting the lateral wall of the nasopharynx to reach the distal lateral portion of the middle cranial fossa requires deep knowledge of the relationship linking various critical structures. To reach this area, the pterygoid process and the pterygoid and soft palate muscles will both have to be removed.

Once the medial pterygoid muscle has been removed, the tensor veli palatini will become visible. It inserts on the basisphenoid, runs from the scaphoid fossa toward the pterygoid uncus, and ends in the soft palate. It is a tiny muscle oriented on a coronal plane and in contact with the cartilaginous portion of the eustachian tube (ET) (Figure 5A). Once removed, the levator veli palatini comes into view: its skull base insertion is level with the petrous apex and ET; it runs inferomedially to the soft palate and is bulkier than the tensor veli palatini.

Once the bony tract of the tube is identified, its cartilaginous portion is removed, and the inferior aspect of the anterior foramen lacerum becomes visible. Located just anterior to the tube, the mandibular nerve $(\mathrm{MN})$ running through the foramen ovale is quite easy to detect. Once the middle meningeal artery (MMA) has been identified, the parapharyngeal portion of the
ICA is isolated posteromedially to the MN and MMA, and posteriorly to the bony ET.

Thanks to a curved blunt probe mimicking the cartilaginous tube, the carrefour among the vidian nerve, MN, MMA, ICA, and the ET can be clearly appreciated (Figures 5B, C). (see Video 5, Supplemental Digital Content 5, http://youtu.be/3GtZdg6Zv6s).

\section{Disclosure}

The authors have no personal, financial, or institutional interest in any of the drugs, materials, or devices described in this article.

\section{Acknowledgments}

Pietro Cardani, Corrado Rispoli, Claudio Valenza, Sara Giordano (Neuromed spa); Alexander Giordano; Rocco and Enzo Reitano (LAREM Studios).

\section{COMMENT}

The authors have provided a series of endonasal cadaveric dissections of the paranasal sinuses, parasellar region, and infratemporal fossa on well prepared and injected cadaver specimens. They demonstrate standard endoscopic corridors and bone removal, with relevant anatomy. The dissections are clear and well narrated. The authors are to be congratulated for a terrific contribution that should become a standard for all residency and skull base fellowship training program curricula.

William T. Couldwell Salt Lake City, Utah 